\title{
Abundances of planetary nebulae in the Galactic bulge ${ }^{\star}$
}

\author{
S. R. Pottasch ${ }^{1}$ and J. Bernard-Salas ${ }^{2}$ \\ ${ }^{1}$ Kapteyn Astronomical Institute, PO Box 800, 9700 AV Groningen, The Netherlands \\ e-mail: pottasch@astro.rug.nl \\ 2 The Open University, MK7 6AA Milton Keynes, UK
}

Received 20 November 2014 / Accepted 10 August 2015

\section{ABSTRACT}

\begin{abstract}
Context. Planetary nebulae (PNe) abundances are poorly known for those nebulae in the Galactic bulge. This is because of the high and uneven extinction in the bulge which makes visual spectral measurements difficult. In addition, the extinction corrections may be unreliable. Elements considered are $\mathrm{O}, \mathrm{N}, \mathrm{Ne}, \mathrm{S}, \mathrm{Ar}$, and $\mathrm{Cl}$.

Aims. We determine the abundances in $19 \mathrm{PNe}, 18$ of which are located in the bulge. This doubles the number of PNe abundance determinations in the bulge. The Galactic abundance gradient is discussed for five elements.

Methods. The mid-infrared spectra measured by the Spitzer Space Telescope are used to determine the abundances. This part of the spectrum is little affected by extinction for which an uncertain correction is no longer necessary. In addition the connection with the visible and ultraviolet spectrum becomes simpler because hydrogen lines are observed both in the infrared and in the visible spectra. In this way we more than double the number of PNe with reliable abundances.

Results. Reliable abundances are obtained for O, N, Ne, S, and Ar for Galactic bulge PNe.

Conclusions. The Galactic abundance gradient is less steep than previously thought. This is especially true for oxygen. The sulfur abundance is reliable because all stages of ionization expected have been measured. It is not systematically low compared to oxygen as has been found for some Galactic PNe.
\end{abstract}

Key words. planetary nebulae: general - ISM: abundances - Galaxy: bulge - infrared: ISM

\section{Introduction}

The study of chemical abundance in the Galaxy is a study of the evolution that is taking place. An important part of the study is the determination of the abundances as a function of position in the Galaxy. A large step forward was made in the work of Shaver et al. (1983) who studied abundances in H II regions throughout much of the Galaxy. The choice of H II regions was based on the fact that emission line objects are much easier to see at large distances, although the abundance gradients they found did not extend into the Galactic bulge.

In the work that followed much use was made of abundances in planetary nebulae (PNe). Like H II regions their line emissions makes them relatively easy to see at large distances, although their precise distance is often difficult to determine. Many abundances are based on spectra taken in the visual region with different telescopes and dispersions. Those that include bulge $\mathrm{PNe}$ and will be discussed here are studies of Webster (1988), Ratag (1990), Cuisinier et al. (2000), Escudero et al. (2004), Exter et al. (2004), Gorny et al. (2004, 2009, 2010), Wang \& Liu (2007), Chiappini et al. (2009). Several of these papers are from the same group.

The abundances found by these authors are quite often uncertain, especially for the bulge PNe. This can be seen by comparing the abundances for the same nebula given by different authors (and ourselves). In every case we consider, at least one and

\footnotetext{
* Based on observations with the Spitzer Space Telescope, which is operated by the Jet Propulsion Laboratory, California Institute of Technology.
}

usually several elements differ in abundance by at least a factor of two and in $25 \%$ of the cases by a factor of 10 . There are several reasons for this. One is that there is a large extinction in the direction of most bulge $\mathrm{PNe}$ which is difficult to measure accurately. This can be seen in the different values given by the different authors (e.g. Pottasch \& Bernard-Salas 2013). The different values of extinction lead not only to different line intensities, but more importantly to different electron temperatures which can strongly affect the abundances determined. Another reason for the different abundances is the correction that is made for the missing stages of ionization. This is often difficult to determine and, as we shall see, has especially important effects on Ar, S, and $\mathrm{Cl}$, but sometimes on $\mathrm{O}$ and $\mathrm{N}$ as well.

Both of these difficulties can be corrected by using the midinfrared spectra measured by the Spitzer Space Telescope. A limited number of bulge PNe have already been analysed in this way by Gutenkunst et al. (2008). These measurements include hydrogen lines, which are essentially unaffected by extinction. Combining these hydrogen lines with the measurement of $\mathrm{H} \beta$ gives a more accurate value of extinction than the difficultto-measure Balmer decrement. Not only does this increase the accuracy of the electron temperature $\left(T_{\mathrm{e}}\right)$ from the visible lines of [N II] and [O III], but lines of as many as three other elements can be measured, which also allow the determination of $T_{\mathrm{e}}$. These are [S III] $(\lambda 6312 / \lambda 18.7 \mu \mathrm{m}),[\mathrm{Ne}$ III] $(\lambda 3869 / \lambda 15.5 \mu \mathrm{m})$, and $[$ Ar III] $(\lambda 7135 / \lambda 8.99 \mu \mathrm{m})$. With a maximum of five independent electron temperature measurements derived from ions with different ionization potential, it is possible to discuss a temperature gradient in the PNe, which may improve the abundance determination. 
Table 1. $\mathrm{H} \beta$ values predicted by infrared hydrogen line and consequent values of $C_{\mathrm{ir}}$.

\begin{tabular}{|c|c|c|c|c|c|c|c|c|c|c|c|}
\hline $\begin{array}{l}\text { Nebula } \\
\text { PNG }\end{array}$ & $\begin{array}{r}\text { Nebula } \\
\text { name }\end{array}$ & $\begin{array}{c}\text { Vel. } \\
\mathrm{km} \mathrm{s}^{-1}\end{array}$ & $\begin{array}{l}\text { Diam. } \\
\text { " }\end{array}$ & $\begin{array}{l}I(7.46)^{\sharp} \\
\left(\times 10^{-14}\right)\end{array}$ & $\begin{array}{c}I(12.37)^{\sharp} \\
\left(\times 10^{-14}\right)\end{array}$ & $\begin{array}{l}T_{\mathrm{e}} \\
\mathrm{K}\end{array}$ & $\begin{array}{c}-\log \mathrm{H} \beta^{\sharp} \\
\mathrm{H}(6-5)\end{array}$ & $\begin{array}{c}-\log \mathrm{H} \beta^{\sharp} \\
\mathrm{H}(7-6)\end{array}$ & $\begin{array}{c}\text { Predicted } \\
-\log H \beta^{\dagger, \#}\end{array}$ & $\begin{array}{c}\text { Measured } \\
-\log \mathrm{H} \beta\end{array}$ & $C_{\mathrm{ir}}$ \\
\hline $000.0-6.8$ & H1-62 & -84 & 1.5 & 18.8 & 13.1 & 6000 & 11.31 & 11.00 & 11.05 & 11.99 & 0.94 \\
\hline $000.7+3.2$ & He2-250 & -175 & 5.0 & 15.0 & 5.80 & 8500 & 11.35 & 11.29 & 11.21 & 13.42 & 2.21 \\
\hline $000.7+4.7$ & $\mathrm{H} 2-11$ & +40 & 2.0 & 38.9 & 16.4 & 8000 & 10.89 & 10.80 & 10.82 & 13.94 & 3.12 \\
\hline $001.2+2.1$ & He2-262 & -172 & 2.32 & 25.7 & 14.4 & 9300 & 11.10 & 10.88 & 10.91 & 13.73 & 2.78 \\
\hline $001.4+5.3$ & H1-15 & +42 & 4.4 & 20.0 & 7.17 & 8500 & 11.20 & 11.19 & 11.19 & 12.70 & 1.51 \\
\hline $001.7-4.6$ & H1-56 & -134 & 2.5 & $22:$ & $8.8:$ & 8500 & 11.19 & 11.11 & 11.16 & 12.14 & 0.98 \\
\hline $002.4-3.7$ & M1-38 & -83 & 3.5 & $46:$ & 16.5 & 7000 & 10.87 & 10.85 & 10.86 & 11.98 & 1.12 \\
\hline $002.6-3.4$ & M1-37 & +202 & 4.33 & & 17 & 6000 & & 10.85 & 10.85 & 12.07 & 1.22 \\
\hline $002.8+1.7$ & $\mathrm{H} 2-20$ & +164 & 3.89 & 31.2 & 11.2 & 7000 & 11.02 & 10.96 & 10.99 & 13.48 & 2.49 \\
\hline $004.9-4.9$ & M1-44 & -107 & 5.7 & & 17.7 & 6400 & & 10.86 & 10.86 & 12.03 & 1.17 \\
\hline $006.0-3.6$ & M2-31 & +136 & 5.1 & 64.0 & 18.5 & 9600 & 10.67 & 10.75 & 10.73 & 12.19 & 1.46 \\
\hline $008.2+6.8$ & $\mathrm{He} 2-260$ & +22 & 1.93 & 23.1 & 8.45 & 11000 & 11.10 & 11.05 & 11.07 & 12.13 & 1.06 \\
\hline $351.2+5.2$ & M2-5 & -128 & 5.0 & 28.0 & 8.1 & 6000 & 11.02 & 11.19 & 11.10 & 12.10 & 1.00 \\
\hline $354.2+4.3$ & M2-10 & -75 & 4.0 & 19.7 & 7.85 & 6750 & 11.26 & 11.13 & 11.19 & 12.62 & 1.53 \\
\hline $356.5-2.3$ & M1-27 & -16.5 & 6.6 & 151 & 56.0 & 6700 & 10.40 & 10.43 & 10.42 & 12.23 & 1.81 \\
\hline $357.1-4.7$ & H1-43 & +76 & 3.0 & $15.5^{:}$ & 10.1 & 6000 & 11.39: & 11.11 & 11.16 & 12.52 & 1.36 \\
\hline $358.9+3.2$ & H1-20 & +190 & 4.0 & 41.8 & 13.0 & 8500 & 10.90 & 10.93 & 10.91 & 13.03 & 2.12 \\
\hline $358.9-3.7$ & H1-44 & +80 & 3.6 & & 6.60 & 6000 & & 11.29 & 11.29 & 13.24 & 1.95 \\
\hline $359.3-1.8$ & M3-44 & -89 & 4.0 & 51 & 17 & 6000 & 10.88 & 10.52 & 10.55 & 13.92 & 3.37 \\
\hline
\end{tabular}

Notes. ${ }^{(\dagger)}$ The $\mathrm{H} \beta$ flux is found from the infrared hydrogen lines using the theoretical ratios of Hummer \& Storey (1987). It must be remembered that the $12.37 \mu \mathrm{m}$ line is a blend of the $\mathrm{H}(7-6)$ transition with the $\mathrm{H}(11-8)$ line, while the $7.46 \mu \mathrm{m}$ line is a blend of $\mathrm{H}(6-5)$ with $\mathrm{H}(8-6)$ and $\mathrm{H}(17-8)$. These blends are subtracted when deriving $\mathrm{H} \beta$. Furthermore the ratios of the various hydrogen lines are (weakly) dependent on the electron temperature. ${ }^{(\sharp)}$ Units of line intensity are always erg $\mathrm{cm}^{-2} \mathrm{~s}^{-1}$. (:) Denotes uncertain value.

A further advantage of the mid-infrared spectra is in assessing the correct ionization correction factor (ICF). Using only the visual spectra, almost all of the elements concerned (except oxygen) are represented only by a single ion.Very often this ion is not the most abundant ion and an ICF must be used, but the ICF is very difficult to determine accurately and, as we will show, large errors are sometimes made. The mid-infrared spectrum often contains additional ions of the element in question. This is true of $\mathrm{Ar}$ ([Ar II] in addition to [Ar III]), S ([S III] and [S IV] in addition to [S II]), and $\mathrm{Ne}$ ([Ne II] in addition to [Ne III]). Usually these ions dominate the contribution to the total abundance for these elements.

In this paper we will discuss 19 Galactic bulge PNe which have both visual and mid-infrared Spitzer spectra. From these spectra we will discuss their extinction, their electron temperature and density and the abundance of the elements $\mathrm{He}, \mathrm{O}, \mathrm{N}$, $\mathrm{Ne}, \mathrm{S}, \mathrm{Ar}$ and sometimes $\mathrm{Cl}$. We then compare these abundances with those literature values found from the visual spectrum and show that often a large difference exists. Of these $19 \mathrm{PNe}, 9$ have been discussed already by Gutenkunst et al. (2008). We use the same line intensities which they measure in the infrared, but we make use of improved electron temperature and density. Their values of $T_{\mathrm{e}}$ were taken from the literature (visual spectral determinations) while we take advantage of the extra temperature determinations made possible in Ar, S and sometimes Ne. These new values only affect the oxygen abundance, and that only to a limited extent. Gutenkunst et al. (2008) find that the bulge abundances do not follow the trend of increasing abundance values closer toward the bulge and we are able to confirm this result.

In Sect. 2 the Spitzer mid-infrared observations are presented and discussed. The results are given in Table 1. In Sect. 3 the values of $T_{\mathrm{e}}$ and electron density $\left(n_{\mathrm{e}}\right)$ are derived and listed in
Table 2. Using these values of $T_{\mathrm{e}}$ and $n_{\mathrm{e}}$ together with both the mid-infrared and visual line intensities, the abundances are then given in Sect. 4. Many of the abundances derived earlier from only the visual spectrum have large differences with those presented here (see references listed above). Finally in Sect. 5 the Galactic bulge abundances are summarized, compared with earlier discussions as well as with the abundance gradients of these elements found by others in the Galactic disc.

\section{The infrared spectrum}

\subsection{Observations}

Of the 19 PNe measured, 17 are almost certainly in the Galactic bulge. They are all within $10^{\circ}$ of the Galactic centre, with two exceptions they have low intrinsic $6 \mathrm{~cm}$ radio continuum flux density (below $30 \mathrm{mJy}$ ), and all but two have very high velocity with respect to the Sun. The observations were made using the Infrared Spectrograph (IRS, Houck et al. 2004) on board the Spitzer Space Telescope with AOR keys both on target and on the background. The basic steps in the reduction of the spectra follow that of Gutenkunst et al. (2008). The only difference is that we start from the basic calibrated data as opposed to the droop images (which lack flatfielding) used in Gutenkunst et al. (2008), and the use of optimal extraction (Lebouteiller et al. 2010) for the low resolution modules for PNe with diameters lower than $4^{\prime \prime}$.

Two of the three diaphragms used have high resolution: the short high module ( $\mathrm{SH}$ ) measures from $9.9 \mu \mathrm{m}$ to $19.6 \mu \mathrm{m}$ and the long high module (LH) from $18.7 \mu \mathrm{m}$ to $37.2 \mu \mathrm{m}$. The $\mathrm{SH}$ has a diaphragm size of $4.7^{\prime \prime} \times 11.3^{\prime \prime}$, while the LH is $11.1^{\prime \prime} \times$ $22.3^{\prime \prime}$. The diameters of the PNe being measured are shown in 
S. R. Pottasch and J. Bernard-Salas: Abundances in the Galactic bulge

Table 2. Mid-infrared line intensities measured by the IRS in units of $10^{-14} \mathrm{erg} \mathrm{cm}^{-2} \mathrm{~s}^{-1}$.

\begin{tabular}{|c|c|c|c|c|c|c|c|c|c|c|c|c|c|}
\hline $\begin{array}{l}\text { Nebula } \\
\text { PNG }\end{array}$ & $\begin{array}{r}6.98 \\
\text { [Ar II] }\end{array}$ & $\begin{array}{c}8.99 \\
\text { [Ar III] }\end{array}$ & $\begin{array}{c}10.5 \\
{[S \text { IV }]}\end{array}$ & $\begin{array}{c}11.3 \\
{[\mathrm{Cl} \mathrm{I}]} \\
\end{array}$ & $\begin{array}{c}12.8 \\
{[\mathrm{Ne} \mathrm{II}]}\end{array}$ & $\begin{array}{c}14.4 \\
{[\mathrm{Cl} \mathrm{II}]}\end{array}$ & $\begin{array}{c}15.5 \\
{[\mathrm{Ne} \text { III] }}\end{array}$ & $\begin{array}{c}18.7 \\
{[\mathrm{~S} \text { III] }}\end{array}$ & $\begin{array}{c}21.8 \\
{[\text { Ar III] }}\end{array}$ & $\begin{array}{c}25.8 \\
{[\mathrm{O} \text { IV }]}\end{array}$ & $\begin{array}{c}33.5 \\
{[\mathrm{~S} \text { III }]}\end{array}$ & $\begin{array}{c}34.8 \\
{[\mathrm{Si} \mathrm{II}]}\end{array}$ & $\begin{array}{c}36.0 \\
{[\mathrm{Ne} \text { III }]}\end{array}$ \\
\hline $000.0-6.8$ & 354. & 19.7 & & 4.6 & 1150 & 8.6 & & 267 & & & 93.0 & & \\
\hline $000.7+3.2$ & 27.4 & 199 & 1700 & & 95.5 & & 1705 & 455 & 11.8 & 3820 & 361 & & 189 \\
\hline $000.7+4.7$ & 12.1 & 53.3 & 24.8 & & 161 & & 162 & 57.5 & 2.5 & & 11.4 & & 12.1 \\
\hline $001.2+2.1$ & & 160 & 733 & & 148 & & 1690 & 386 & 8.85 & & 206 & & 155 \\
\hline $001.4+5.3$ & 6.84 & 104 & 77.4 & & 432 & & 352 & 354 & 7.35 & & 169 & & \\
\hline $001.7-4.6$ & & 95.5 & 405 & & 65.0 & & 855 & 213 & 6.14 & & 105 & & 61.6 \\
\hline $002.4-3.7$ & 427 & 18.9 & & & 1475 & & & 278 & & & 97.5 & & \\
\hline 002.6-3.4 & 470 & 9.9 & & & 1000 & & & 229 & & 4.0: & 76.0 & & \\
\hline $002.8+1.7$ & 253 & 109 & 10.4 & & 1189 & 11.8 & 17.8 & 601 & 12.4 & & 227 & & \\
\hline $004.9-4.9$ & 337 & 65.0 & 23.0 & & 975 & & 281 & 624 & 4.4: & & 270 & 73.7 & 41.2: \\
\hline $006.0-3.6$ & 36.0 & 304 & 2480 & & 113 & & 3680 & 704 & 21.5 & 17.4 & 218 & & 292 \\
\hline $008.2+6.8$ & 60.4 & & & & 286.0 & & & 43.7 & & 5.0: & 12.3: & 12.6 & \\
\hline $351.2+5.2$ & 305 & 174 & 19.8 & & 1132 & & 126 & 795 & 9.27 & & 499 & & \\
\hline $354.2+4.3$ & 92.8 & 193 & 183 & & 688 & & 676 & 600 & 11.8 & & 418 & & 70.9 \\
\hline $356.5-2.3$ & 1600 & 56.8 & & 23.4 & 5430 & 25.8 & & 1010 & & & 310 & & \\
\hline $357.1-4.7$ & 246 & & & & 580 & 5.1 & & 23.9 & & & & & \\
\hline $358.9+3.2$ & 90.0 & 586 & 1960 & & 249 & & 5640 & 945 & 37.4 & 108 & 278 & & 415 \\
\hline $358.9-3.7$ & 167 & 199 & 86.0 & & 821 & & 369 & 926 & 12.6 & & 290 & 44.0 & 27.0 \\
\hline $359.3-1.8$ & 882 & 16.0 & & 17 & 2790 & 21 & & 590 & & & 164 & & \\
\hline
\end{tabular}

the fourth column of Table 1. These are taken from a variety of sources, the most reliable are those of Sahai et al. (2011). For the other PNe the VLA radio continuum half width measurements listed by Acker et al. (1992) are given. The diameters are always considerably smaller than the LH diaphragm but may not always be smaller than the SH diaphragm. To correct for possible missing intensity in the $\mathrm{SH}$ diaphragm we examine the continuum intensity of $\mathrm{LH}$ and $\mathrm{SH}$ in the region of wavelength overlap at $19 \mu \mathrm{m}$. These continua should be equal if the entire nebula is being measured in the SH diaphragm. If this is not so all intensities in the SH diaphragm are increased by a factor which make the continua equal. The corrections are small for these nebulae, usually between 1.1 and 1.3 , indicating that even the smaller diaphragm measured nearly the entire nebula.

The third diaphragm is a long slit which is 4 " wide and extends over the entire nebula. This SL module measures in low resolution in the wavelength region between $5.5 \mu \mathrm{m}$ and $14 \mu \mathrm{m}$. These spectra are normalized by making the strong lines in common between the SL and SH modules agree $(10-14.5 \mu \mathrm{m})$. Especially important is the agreement of the [S IV] line at $10.51 \mu \mathrm{m}$ and the [Ne II] line at $12.82 \mu \mathrm{m}$. Because all of the PNe measured are small, the corrections are likewise small. The resultant combined spectra may now be plotted. We have not done this here because good plots are already in the literature. Plots of He2-250, H2-11, He2-262, H1-15, H2-20, M2-31, M2-5, M2-10 and H1-20 may be found in Gutenkunst et al. (2008); plots of He2-260, M1-27, H1-43 and M3-44 can be found in Perea-Calderon et al. (2009); plots of M1-38 and M1-37 are found in Stanghellini et al. (2012). The excellent quality of the emission lines is apparent from the spectra.

It is also necessary to measure the infrared hydrogen lines because the ratio of these lines to $\mathrm{H} \beta$ is the most reliable way of obtaining the extinction. The intensities of the $12.37 \mu \mathrm{m}$ hydrogen line (SH diaphragm) and the $7.46 \mu \mathrm{m}$ line (SL diaphragm)
Table 3. Additional mid-infrared line intensities measured by IRS in units of $10^{-14} \mathrm{erg} \mathrm{cm}^{-2} \mathrm{~s}^{-1}$.

\begin{tabular}{|c|c|c|c|c|c|c|}
\hline $\begin{array}{l}\text { Nebula } \\
\text { PNG }\end{array}$ & $\begin{array}{r}7.90 \\
{[\mathrm{Arv}]}\end{array}$ & $\begin{array}{c}13.1 \\
{[\mathrm{Arv}]}\end{array}$ & $\begin{array}{c}14.3 \\
{[\mathrm{Ne} \mathrm{V}]}\end{array}$ & $\begin{array}{c}22.9 \\
{[\mathrm{Fe}} \\
\mathrm{III}]\end{array}$ & $\begin{array}{c}24.3 \\
{[\mathrm{Ne} \mathrm{V}]}\end{array}$ & $\begin{array}{c}25.9 \\
{[\mathrm{Fe} \text { II }]}\end{array}$ \\
\hline $000.7+3.2$ & 10.1 & 16.2 & 23.9 & & 32.3 & \\
\hline $002.6-3.4$ & & & & & & $3.3:$ \\
\hline $008.2+6.8$ & & & & 15.3 & & 10.9 \\
\hline $356.5-2.3$ & & & & 26.3 & & \\
\hline $358.9-3.7$ & & & & & & 3.7 \\
\hline
\end{tabular}

Notes. (:) Denotes uncertain value.

are then measured using the gaussian line-fitting routine. The resultant values are shown in Cols. 5 and 6 of Table 1 . The $3 \sigma$ errors of measurement are always less than $20 \%$. The $12.37 \mu \mathrm{m}$ line has the more accurate intensity of the two lines although it is intrinsically the weaker line. There are several reasons for this. First of all the $12.37 \mu \mathrm{m}$ line is measured with a much higher resolution (about 600) compared to the $7.46 \mu \mathrm{m}$ line (about 90). Secondly the measurement of the $7.46 \mu \mathrm{m}$ line is difficult because it falls in the middle of overlapping orders. Thirdly the $12.37 \mu \mathrm{m}$ line is often measured both at high resolution (SH) and at low resolution (SL) and very similar intensities are found. For these reasons we give the value of the intrinsic $\mathrm{H} \beta$ found from the $12.37 \mu \mathrm{m}$ line considerably more weight. The intensities given in the table include a correction for extinction, which is quite small at these infrared wavelengths.

The majority of the measured line intensities are listed in Tables 2, while Table 3 lists additional lines detected in only a few objects. Just as in Table 1 a correction is made for the small extinction present. 
Table 4. Electron temperatures (in K) computed from different ions.

\begin{tabular}{l|cccccc}
\hline \hline $\begin{array}{l}\text { Nebula } \\
\text { PNG }\end{array}$ & $\begin{array}{c}T_{\mathrm{e}} \\
{[\mathrm{N} \mathrm{II}]}\end{array}$ & $\begin{array}{c}T_{\mathrm{e}} \\
{[\mathrm{S} \mathrm{III}]}\end{array}$ & $\begin{array}{c}T_{\mathrm{e}} \\
{[\mathrm{Ar} \text { III] }]}\end{array}$ & $\begin{array}{c}T_{\mathrm{e}} \\
{[\mathrm{O} \text { III] }]}\end{array}$ & $\begin{array}{c}T_{\mathrm{e}} \\
{[\mathrm{Ne} \text { III] }]}\end{array}$ & $\begin{array}{c}T_{\mathrm{e}} \\
\text { Ave. }\end{array}$ \\
\hline $000.0-6.8$ & 6200 & 6000 & 5800 & & & 6000 \\
$000.7+3.2$ & 8200 & 8300 & 8700 & 13500 & 7600 & $8500^{*}$ \\
$000.7+4.7$ & 8500 & 8000 & 8000 & 10300 & & $8000^{*}$ \\
$001.2+2.1$ & & 9500 & 9300 & & 8000 & 9000 \\
$001.4+5.3$ & 8500 & 7200 & 8300 & & & 8500 \\
$001.7-4.6$ & 9000 & & 10000 & 7800 & 8400 & 8500 \\
$002.4-3.7$ & 7000 & 6700 & 7000 & & & 7000 \\
$002.6-3.4$ & 5800 & & 6300 & & & 6000 \\
$002.8+1.7$ & 6000 & & 6500 & & & 7000 \\
$004.9-4.9$ & 6400 & & 6300 & & & 6300 \\
$006.0-3.6$ & 8200 & 10200 & 9800 & 9700 & 9500 & 9600 \\
$008.2+6.8$ & 11200 & 10900 & & & & 11000 \\
$351.2+5.3$ & 6000 & & 6000 & & & 6000 \\
$354.2+4.3$ & 7000 & 6700 & 6500 & & 6300 & 6900 \\
$356.5-2.3$ & 6500 & 7500 & 6500 & & & 6700 \\
$357.1-4.7$ & 6000 & & & & & 6000 \\
$358.9+3.2$ & 9000 & 8000 & 8000 & & 7300 & 8500 \\
$358.9-3.7$ & 6200 & & 5900 & & & 6000 \\
$359.3-1.8$ & 6000 & & $8000:$ & & & 6000 \\
\hline
\end{tabular}

Notes. ${ }^{(*)}$ This value of temperature is used for the lower ionization ions. For [O III] and higher ionization potentials a value of 10500 is used.

\section{Electron temperature, density and radiation field}

\subsection{Temperature}

When only the visual spectrum is used, the electron temperature is obtained by the ratios of [O III] $\lambda 4363 \AA / \lambda 5007 \AA$, and [N II] $\lambda 5755 \AA / 6584 \AA$. Because the observed value of $\lambda 5755 \AA$ is often low it is difficult to measure, leading to an uncertain value of electron temperature and consequently to uncertain abundances. In addition there is an uncertainty introduced by a possible temperature gradient in the nebula. These difficulties can be reduced when the mid-infrared spectrum is available because in this case it is possible to find as many as three additional measures of the temperature: [S III] $\lambda 18.7 \mu \mathrm{m} / \lambda 6312 \AA$, [Ar III] $\lambda 8.99 \mu \mathrm{m} / \lambda 7135 \AA$, [Ne III] $\lambda 15.5 \mu \mathrm{m} / \lambda 3869 \AA$.

The resultant temperatures are listed in Table 4 . The values shown are consistent with a common value for most of the nebulae. For PNG 000.7+3.2 and PNG 000.7+4.7 a higher temperature is measured at the inner part of the nebulae from the [O III] and $[\mathrm{Ne}$ III] ions. Note that the [Ne III] temperature is more uncertain than the other values because the correction for extinction is difficult to make at $\lambda 3869 \AA$. The values of $T_{\mathrm{e}}$ which will be used for each PN are listed in the final column of Table 4.

\subsection{Density}

Almost all of the PNe have electron density measured by [S III] $\lambda 18.7 \mu \mathrm{m} / \lambda 33.5 \mu \mathrm{m}$ and by [S II] $\lambda 6731 \AA / \lambda 6717 \AA$. We use the average of these two values for the density of a given PN. All values found are between $10^{3}$ and $10^{4} \mathrm{~cm}^{-3}$. The values of density are not importantly influenced by extinction since the lines used are close in wavelength. There is no clear indication of different densities as a function of radius in any of the nebulae. Only a few cases of determination of density from other ions ([Ar III], [O II], and [Cl III]) have been made. They are in
Table 5. Electron densities $\left(\right.$ in $\left.\mathrm{cm}^{-3}\right)$.

\begin{tabular}{l|cc}
\hline \hline Nebula & $n_{\mathrm{e}}([\mathrm{S} \mathrm{II}])$ & $n_{\mathrm{e}}([\mathrm{S} \mathrm{III}])$ \\
\hline $000.0-6.8$ & 3700 & 3300 \\
$000.7+3.2$ & 1900 & 1000 \\
$000.7+4.7$ & 9500 & 8000 \\
$001.2+2.1$ & 3000 & 1600 \\
$001.4+5.3$ & 950 & 2700 \\
$001.7-4.6$ & 2000 & 3000 \\
$002.4-3.7$ & 4200 & 5000 \\
$002.6-3.4$ & 4300 & 3200 \\
$002.8+1.7$ & 4800 & 3000 \\
$004.9-4.9$ & 1800 & 2500 \\
$006.0-3.6$ & 5200 & 4000 \\
$008.2+6.8$ & 17000 & 5800 \\
$351.2+5.3$ & 1500 & 1600 \\
$354.2+4.3$ & 1400 & 1500 \\
$356.5-2.3$ & 4000 & 3800 \\
$357.1-4.7$ & 6500 & \\
$358.9+3.2$ & 4400 & 5000 \\
$358.9-3.7$ & 5000 & 6000 \\
$359.3-1.8$ & 2000 & 3000 \\
\hline
\end{tabular}

reasonable agreement with the $[\mathrm{S} \mathrm{II}]$ and $[\mathrm{S} \mathrm{III}]$ densities listed in Table 5 .

\subsection{Radiation field}

The distribution of radiation originating in the central star can be studied by its effect on the ionization in the nebula. For several of the nebulae several stages of ionization of a single element are measured, which contains information about the spectrum of the exciting star. In the infrared these stages are $\mathrm{Ne}^{++} / \mathrm{Ne}^{+}, \mathrm{Ar}^{++} / \mathrm{Ar}^{+}$and $\mathrm{S}^{+3} / \mathrm{S}^{++}$. Unfortunately this cannot be interpreted directly as the temperature of the exciting star because other parameters also play a role in determining these ratios. Among others these are the size of the star, the size of the nebula, the abundances in the nebula and the departure from a blackbody of the stellar spectrum. In principle the spectrum may be determined from the above ratios by making a model of the star-nebula combination. Because the model is dependant on so many imperfectly known parameters it is difficult to make an accurate model. This can be seen in the models published by Surendiranath \& Pottasch (2008) and Surendiranath et al. (2004) where the best-fitting models do not accurately reproduce the above ratios. See also the discussion of this problem by Mokiem et al. (2004). The observed ratios do permit deriving approximate stellar temperatures. For those nebulae for which the line ratio $15.5 / 12.8$ ([Ne III $] /[\mathrm{Ne} \mathrm{II}])$ is greater than 10 , the stellar temperature is greater than $80000 \mathrm{~K}$, sometimes much greater. On the other hand, when the line ratio 8.99/6.98 ([Ar III]/[Ar II]) is less than 0.2 , the stellar temperature is less than $35000 \mathrm{~K}$. To obtain more precise values of the temperature it is necessary to model the star-nebula combination which can be a difficult task.

\section{PNe abundances}

Knowing the electron temperature and density, the PNe abundances may be computed using both the infrared spectrum presented here together with the visual spectrum found in the literature. 
Table 6. Observed (measured) ionic abundances ${ }^{\dagger}$ with respect to hydrogen.

\begin{tabular}{l|rcc|cc|ccc|ccc|cc}
\hline \hline $\begin{array}{l}\text { Nebula } \\
\text { PNG }\end{array}$ & $\begin{array}{r}\mathrm{O}^{+} \\
(-4)\end{array}$ & $\begin{array}{c}\mathrm{O}^{++} \\
(-4)\end{array}$ & $\begin{array}{c}\mathrm{O}^{+3} \\
(-4)\end{array}$ & $\begin{array}{c}\mathrm{Ne}^{+} \\
(-4)\end{array}$ & $\begin{array}{c}\mathrm{Ne}^{++} \\
(-4)\end{array}$ & $\begin{array}{c}\mathrm{S}^{+} \\
(-6)\end{array}$ & $\begin{array}{c}\mathrm{S}^{++} \\
(-6)\end{array}$ & $\begin{array}{c}\mathrm{S}^{+3} \\
(-6)\end{array}$ & $\begin{array}{c}\mathrm{Ar}^{+} \\
(-6)\end{array}$ & $\begin{array}{c}\mathrm{Ar}^{++} \\
(-6)\end{array}$ & $\begin{array}{c}\mathrm{Ar}^{+3} \\
(-6)\end{array}$ & $\begin{array}{c}\mathrm{Cl}^{+} \\
(-7)\end{array}$ & $\begin{array}{c}\mathrm{Cl}^{++} \\
(-7)\end{array}$ \\
\hline $000.0-6.8$ & 3.7 & - & - & 3.08 & - & 5.9 & 4.4 & - & 6.85 & 0.30 & - & 3.0 & - \\
$000.7+3.2$ & 1.6 & 1.76 & 2.85 & 0.35 & 2.48 & 0.20 & 10.9 & 10.3 & 0.75 & 4.5 & 0.12 & - & - \\
$000.7+4.7$ & 2.2 & 1.5 & - & 2.2 & 0.96 & 1.26 & 7.90 & 0.89 & 1.28 & 4.4 & - & - & - \\
$001.2+2.1$ & 1.2 & 3.9 & - & 0.22 & 1.05 & 0.27 & 3.84 & 1.93 & - & 1.6 & - & - & - \\
$001.4+5.3$ & 2.3 & 1.8 & - & 1.1 & 0.39 & 0.18 & 5.61 & 0.31 & 0.14 & 1.8 & - & - & - \\
$001.7-4.6$ & 0.32 & 4.0 & - & 0.15 & 0.82 & 0.11 & 3.42 & 1.71 & - & 1.4 & 0.010 & - & 0.96 \\
$002.4-3.7$ & 4.2 & 0.014 & - & 2.5 & - & 3.2 & 2.9 & - & 5.3 & 0.19 & - & 2.66 & - \\
$002.6-3.4$ & 1.9 & 0.057 & 0.0015 & 1.5 & - & 4.0 & 2.7 & - & 5.3 & 0.11 & - & - & - \\
$002.8+1.7$ & 3.7 & 0.38 & - & 2.7 & 0.016 & 1.8 & 8.7 & 0.035 & 4.3 & 1.4 & - & - & - \\
$004.9-4.9$ & 1.6 & 0.24 & - & 1.65 & 0.17 & 3.7 & 6.8 & 0.066 & 4.46 & 0.67 & - & - & - \\
$006.0-3.6$ & 0.40 & 4.7 & 0.003 & 0.015 & 1.3 & 0.46 & 3.9 & 3.7 & 0.23 & 1.7 & - & - & 1.25 \\
$008.2+6.8$ & 1.3 & 0.013 & 0.005 & 0.61 & - & 0.33 & 1.1 & - & 1.0 & 0.05 & - & - & - \\
$351.2+5.3$ & 3.7 & 0.66 & - & 4.0 & 0.16 & 2.52 & 15.4 & 0.11 & 7.55 & 2.7 & - & - & - \\
$354.2+4.3$ & 3.0 & 2.1 & - & 2.5 & 0.91 & 3.4 & 12.5 & 1.1 & 2.5 & 3.5 & - & - & 2.1 \\
$356.5-2.3$ & 4.6 & - & - & 3.0 & - & 3.54 & 3.68 & - & 6.9 & 0.19 & - & 5.4 & - \\
$357.1-4.7$ & 1.5 & 0.066 & - & 1.05 & - & 4.3 & 6.2 & - & 3.44 & - & - & 1.4 & - \\
$358.9+3.2$ & 1.75 & 6.4 & 0.032 & 0.37 & 3.26 & 1.16 & 8.1 & 4.7 & 0.97 & 5.2 & - & - & 2.0 \\
$358.9-3.7$ & $8.75:$ & 2.91 & - & 1.62 & 0.62 & 3.66 & 12.8 & 0.74 & 4.7 & 5.3 & - & - & $28:$ \\
$359.3-1.8$ & $6.5:$ & - & - & 2.4 & - & 6.15 & 3.08 & - & 5.7 & 0.08 & - & 2.4 & - \\
\hline
\end{tabular}

Notes. $\left.{ }^{\dagger}\right)$ In addition to these, $\mathrm{Fe}^{+}$has been measured in PNG 2.6-3.4 $\left(0.91 \times 10^{-7}\right)$, in PNG 8.2+6.8 $\left(5.4 \times 10^{-7}\right)$ and in PNG $358.9-3.7(2.3 \times$ $\left.10^{-7}\right) ; \mathrm{Fe}^{++}$in PNG 8.2+6.8 $\left(7.5 \times 10^{-7}\right)$ and $\mathrm{Si}^{+}$in PNG 8.2+6.8 $\left(2.9 \times 10^{-7}\right)$ and PNG 358.9-3.7 $\left(7.7 \times 10^{-6}\right)$.

\subsection{Ionic abundances}

References to the visual spectra are given in the introduction (second paragraph). We use an average value for individual lines, giving more weight to those measurements where ions observed in both the infrared and visual spectra give very similar results. The visual spectrum is important in determining the abundances of [O II], [O III], [N II], [S II] and [Cl II] while the infrared spectrum is used for determining the abundances of [S III], [S IV], [Ne II], [Ne III], [Ne V], [Ar II] and [Ar III]. The resultant ionic abundances are shown in Table 6 . The abundances found from the infrared lines are probably more reliable because they are not very dependant on the electron temperature. In addition, for $\mathrm{Ne}, \mathrm{S}$, and Ar all the important ionization stages are measured so that no corrections for missing stages of ionization need be made. Infrared measurements are not very dependant on knowledge of the extinction. Thus the errors in this case are simply due to errors in the intensity measured for the line in question which we estimate to be about $20 \%$. For the abundance found from the visual lines we estimate the error to be twice as large. Only values of oxygen which are determined from [O III] are better determined because the [O III] lines, being close to $\mathrm{H} \beta$ can be more easily corrected for extinction.

In Table 6 the abundances of the individual ions are given in those cases in which at least one line belonging to that ion has been measured. The ions of $\mathrm{He}$ and $\mathrm{N}$ are not given. In the case of nitrogen this is because only one ion of this element has been measured, $\mathrm{N}^{+}$. It has been assumed that only one additional nitrogen ion is present $\left(\mathrm{N}^{++}\right)$and its abundance can be found from $\mathrm{N}^{++}=\mathrm{N}^{+}\left(\mathrm{O}^{++} / \mathrm{O}^{+}\right)$(e.g. Kingsburgh \& Barlow 1994). The resultant sum of the two ions is listed in Table 7 as the total $\mathrm{N}$ abundance. In the case of helium the value listed in Table 7 refers to $\mathrm{He}^{+}$with occasionally a small contribution of $\mathrm{He}^{++}$.
Table 7. Abundances with respect to hydrogen.

\begin{tabular}{l|rcccccc}
\hline \hline Nebula & $\mathrm{He}$ & $\begin{array}{c}\mathrm{O} \\
\text { PNG }\end{array}$ & $\begin{array}{c}\mathrm{N} \\
(-4)\end{array}$ & $\begin{array}{c}\mathrm{Ne} \\
(-4)\end{array}$ & $\begin{array}{c}\mathrm{S} \\
(-6)\end{array}$ & $\begin{array}{c}\mathrm{Ar} \\
(-6)\end{array}$ & $\begin{array}{c}\mathrm{Cl} \\
(-7)\end{array}$ \\
\hline $000.0-6.8$ & 0.010 & 3.8 & 1.65 & 3.1 & 11. & 7.2 & 3.0 \\
$000.7+3.2$ & 0.15 & 6.4 & 3.2 & 3.0 & 22. & 5.3 & \\
$000.7+4.7$ & 0.178 & 3.7 & 1.6 & 3.3 & 11.1 & 6.5 & \\
$001.2+2.1$ & 0.11 & 5.1 & 0.36 & 1.3 & 6.2 & 1.8 & \\
$001.4+5.3$ & 0.103 & 4.1 & 0.21 & 1.5 & 6.0 & 2.0 & \\
$001.7-4.6$ & 0.11 & 4.5 & 0.53 & 1.0 & 5.3 & 1.8 & 1.3 \\
$002.4-3.7$ & 0.125 & 3.9 & 1.1 & 2.5 & 6.2 & 5.5 & 5.0 \\
$002.6-3.4$ & 0.0085 & 1.9 & 2.7 & 1.5 & 7.0 & 5.5 & \\
$002.8+1.7$ & 0.075 & 4.1 & 1.8 & 2.8 & 10.5 & 5.8 & \\
$004.9-4.9$ & 0.052 & 1.9 & 1.64 & 1.9 & 11. & 5.2 & \\
$006.0-3.6$ & 0.12 & 5.2 & 1.9 & 1.3 & 7.8 & 2.4 & 1.6 \\
$008.2+6.8$ & 0.0087 & 1.3 & 0.16 & 0.61 & 1.5 & 1.1 & \\
$351.2+5.3$ & 0.076 & 4.4 & 2.8 & 4.2 & 18. & 11. & \\
$354.2+4.3$ & 0.135 & 5.1 & 2.5 & 3.5 & 17. & 6.7 & 2.9 \\
$356.5-2.3$ & 0.0084 & 4.8 & 1.4 & 3.0 & 7.8 & 7.3 & 5.6 \\
$357.1-4.7$ & 0.013 & 2.4 & 2.4 & 1.1 & 11. & 3.6 & 1.5 \\
$358.9+3.2$ & 0.126 & 8.2 & 2.65 & 3.7 & 14. & 6.8 & 2.3 \\
$358.9-3.7$ & 0.098 & 11.0 & 4.0 & 2.4 & 17. & 10. & \\
$359.3-1.8$ & 0.011 & 7. & 2.2 & 2.5 & 9.3 & 5.8 & 2,4 \\
\hline Ave(bulge) & & 4.2 & 1.8 & 2.3 & 10.6 & 4.6 & 3.0 \\
vis(bulge) & 0.12 & 3.7 & 1.3 & 0.85 & 6.3 & 1.8 & 16.5 \\
PN(7-9 kpc) & 0.115 & 4.7 & 2.0 & 1.66 & 8.5 & 2.9 & \\
Sun & 0.085 & 4.9 & 0.78 & 0.85 & 13.4 & 2.5 & 3.2 \\
H II(0-4 kpc) & & 6.6 & 1.8 & 1.3 & 6.8 & 4.1 & \\
\hline & & & & & & & \\
\hline
\end{tabular}

References. PN(7-9 kpc) average abundance of PNe within 7 to $9 \mathrm{kpc}$ from the Galactic centre Pottasch \& Bernard-Salas (2010); vis(bulge) average $\mathrm{PNe}$ abundance in the bulge as determined using only visual spectra Chiappini et al. (2009); solar abundance Asplund et al. (2009); $\mathrm{H} \mathrm{II}(0-4 \mathrm{kpc})$ average abundance of $\mathrm{H}$ II regions in the Galactic bulge Martín-Hernández et al. (2003), and Rudolph et al. (2006). 
A\&A 583, A71 (2015)
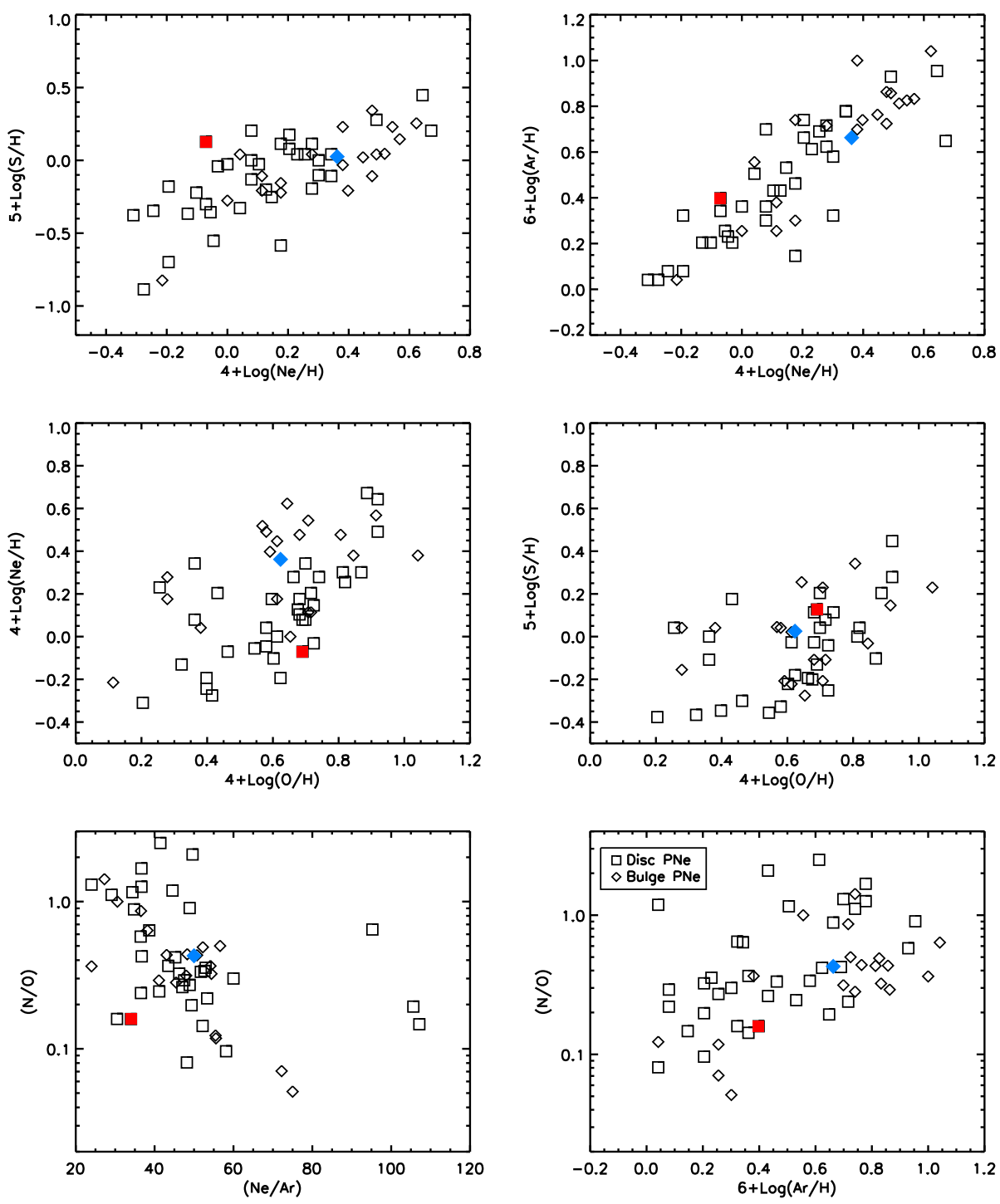

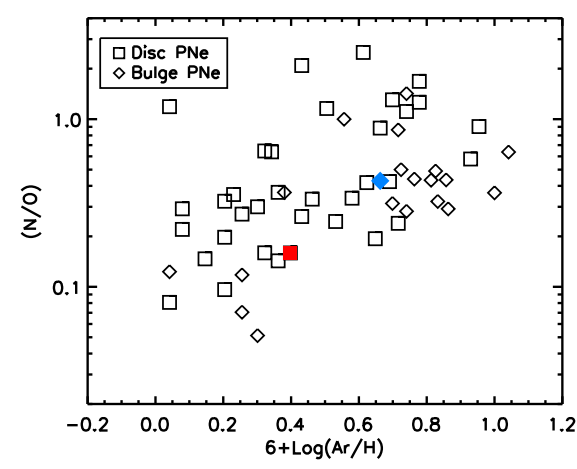

Fig. 1. Abundances of $\mathrm{Ne}, \mathrm{S}, \mathrm{Ar}$ and $\mathrm{O}$ plotted against one another. The bulge PNe are plotted as small squares and the disc PNe as large squares. The red and blue symbols indicate the solar abundances and the average bulge abundances respectively.
There is no way of determining the neutral helium abundance, thus the total helium abundance cannot be determined.

\subsection{Total abundances}

Total abundances are shown in Table 7. Notice that PNG 008.2+6.8 has lower abundances than the other PNe. Except for $\mathrm{He}$ and $\mathrm{N}$ the abundances are remarkably similar to those in Hu1-2 which is in a very different position on the sky. Since PNG008.2+6.8 has a low velocity it may not be a bulge PN.

As mentioned in the introduction, Gutenkunst et al. (2008) has determined the abundances for several of the PNe listed here. Our results agree with theirs within $20 \%$ for all cases in which our abundances are derived from the IR lines. Only in two cases have we found higher oxygen abundances, in PNG 002.8+1.7 and PNG 358.9-3.7 because a lower $T_{\mathrm{e}}$ is used.

In Fig. 1 we plot abundances of several elements ( $\mathrm{Ne}, \mathrm{S}$, $\mathrm{Ar}, \mathrm{O}$, and the $\mathrm{N} / \mathrm{O}$ ratio) against each other. Abundances of the bulge $\mathrm{PNe}$ (shown as diamonds) are from this paper and those of disc PNe (shown as squares) are from Pottasch \& Bernard-Salas (2010). From these diagrams one may note that the abundances of these elements are very similar in the bulge and in the disc. $\mathrm{Ne}$ vs. Ar shows a very good correlation, as does Ne vs. S. This agrees with the expectation that these elements do not change in the course of stellar evolution. While neon enrichment is sometimes possible (Marigo et al. 2003), the good correlation with $\mathrm{Ar}$ and $\mathrm{S}$ indicates that this is not the case for the PNe in this sample. The middle panels in this figure show that there is also a trend for $\mathrm{Ne}$ and Ar to increase with oxygen abundance, albeit with more scatter. This scatter is probably caused by the larger uncertainty in the oxygen abundance because it is derived from the more uncertain optical spectrum, while $\mathrm{Ne}, \mathrm{Ar}$, and $\mathrm{S}$ are derived using mostly IR lines. In addition, in higher mass PNe $\left(>4 M_{\odot}\right.$ ) it is expected that some $\mathrm{O}$ will be converted to $\mathrm{N}$ in the course of evolution during hot bottom burning, which will cause deviations in the abundance relation with $\mathrm{Ne}$ and $\mathrm{S}$. The $\mathrm{N} / \mathrm{O}$ ratio is shown in the last two panels, first plotted vs. Ne/Ar and finally plotted against $\mathrm{Ar} / \mathrm{H}$. The scatter is very high in these two diagrams and it is not clear whether a relation exists.

At the end of Table 7 a comparison of the abundances is made with similar objects. First the average values of the $19 \mathrm{PNe}$ determined above is given. Next the bulge PNe abundances as determined using only the visual spectra are listed. These are taken from the work of Chiappini et al. (2009), and are a merger of the determinations of Gorny et al. (2004, 2009), Wang \& Liu (2007), Cuisinier et al. (2000) and Escudero et al. (2004). These include all of the best bulge PNe measurements. 
The average value of the abundances of the PNe at approximately the solar distance from the centre is given. These are taken from Pottasch \& Bernard-Salas (2010) because these authors included infrared ISO observations. For comparison the Solar abundances are then listed, taken from Asplund et al. (2009) where it should be remembered that although they are listed, neither Ne nor Ar are measured in the Sun. Finally the abundances of $\mathrm{HII}$ regions found in the bulge are given. These are from Martín-Hernández et al. (2003) and are used because they rely on the ISO infrared observations for $\mathrm{N}, \mathrm{Ne}, \mathrm{S}$, and $\mathrm{Ar}$ and thus the more important ions of these elements are included. The abundances are therefore more reliable than other sources. The average include the limited number of measurements within $4 \mathrm{kpc}$ of the Galactic centre. Values of oxygen are taken from Rudolph et al. (2006) and refer to different H II regions than measured by Martín-Hernández et al. (2003).

Consider now the individual elements in individual PNe. The oxygen abundances are probably among the most uncertain because they are only determined from visual lines. Especially the intensities of the [O II] lines are uncertain because the corrections are very sensitive to the extinction used. The $\mathrm{O}^{++}$is better known because the line $\lambda 5007 \AA$ is very close to $\mathrm{H} \beta$, but because of the low level of ionization the $\mathrm{O}^{+}$abundance is dominant in all but three PNe, the oxygen abundance may have an uncertainty of $50 \%$.

The nitrogen abundance is somewhat better determined although only a single stage of ionization is observed. This is because $\mathrm{N}^{+}$is often the dominant stage of ionization. It is generally accepted that much nitrogen is formed in the AGB stage; in this stage the nitrogen abundance increases from a low value, approximately solar, to a much higher value. According to Karakas (2003) and to Marigo et al. (2003) the amount of nitrogen produced depends on the mass of the star; the heavier stars produce much more nitrogen. The $\mathrm{N} / \mathrm{O}$ ratio for the PNe discussed here follow this expectation with $\mathrm{N} / \mathrm{O}$ ratios of between 0.05 and 1.0. The lowest value is somewhat lower than the solar N/O. The fact that the $\mathrm{H}$ II regions in the bulge have a rather high $\mathrm{N} / \mathrm{O}$ ratio means that a substantial number of $\mathrm{PNe}$ have already processed nitrogen and emitted it to the ISM in the bulge.

The neon abundance is well determined in the PNe with infrared measurements. This contrasts strongly with the abundance determined when only optical measures of bulge PNe are available. This has two reasons. First of all in the optical region no [Ne II], often the predominant ion, is observed. Secondly, although a [Ne III] line is observed in the near ultraviolet, its intensity is difficult to determine accurately mainly because correcting for extinction is very uncertain. Thus the present values of bulge PNe neon abundances are unique. The values of neon given in Table 7 are consistently about one half of the value of oxygen in a given nebula and quite well determined. The values are slightly larger than the neon in $\mathrm{PNe}$ at the solar distance from the centre, and from the bulge $\mathrm{H}$ II regions.

We now consider the sulfur abundance. A number of papers have appeared in recent years pointing out that the sulfur abundance compared to oxygen in many $\mathrm{PNe}$ is systematically lower than expected, compared to its value in H II regions or in the sun. A summary of this problem has been given by Henry et al. (2012), who are unable to find a satisfactory explanation for this fact. The sulfur abundances listed in Table 7 for the bulge PNe do not show this anomaly. The sulfur abundance for these PNe does not require an "ionization correction factor" since in the infrared all relevant ionization stages have been measured. It does not appear that the scatter of the sulfur abundances is much higher than that of other elements.
For about half of the PNe the chlorine abundance has been determined. These values are rather uncertain because the lines which they produce are weak and consequentially difficult to measure. For 5 very low ionization PNe an infrared line at $14.3 \mu \mathrm{m}$ due to $\mathrm{Cl}^{+}$has been found. These nebulae are of such low ionization that almost all the chlorine is found in this ionization stage. In 4 additional PNe a very weak line of $\lambda 5537 \AA$ is found which is difficult to measure accurately.

The argon abundance is reasonably accurate because most of the argon is in the form of $\mathrm{Ar}^{+}$and $\mathrm{Ar}^{++}$, both ions being represented by infrared lines. Again there is some scatter in the abundance. The median value, like neon and sulfur, is somewhat higher than the values of these elements at the solar distance from the Galactic centre. This is consistent with a small radial gradient. Unlike Ne and $\mathrm{S}$, the Ar abundance is very similar to the Ar abundance of bulge $\mathrm{H}$ II regions.

\subsection{Comparison with previous determinations}

A comparison can be made between our results, the bulge abundances found from the visual spectra, and the earlier results of Gutenkunst et al. (2008). Consider first the visual spectra. The results are given in the nine papers cited in the introduction. Because the individual results are sometimes very different from each other due to differing measurements leading to different extinction corrections and different electron temperatures, we limit ourselves to a comparison with the very extensive results given by Chiappini et al. (2009). These authors summarize 170 bulge PNe measurements including many earlier results of other authors. These are probably the best bulge PNe abundances determined using only visual spectra and are listed as "vis(bulge)" in Table 7. These authors estimate that the main source of error in their abundances is due to the difficulty of estimating the ICF. After removing 23 low ionization nebulae from their sample, they estimate there is an uncertainty of about $30 \%$ in the abundances they give. An exception is made for sulfur which is thought to have twice as large an error. They have assumed that other sources of error, such as error of measurement and incorrect correction of the extinction, are negligible.

The errors for the abundances we have found, as discussed above, do not depend on the (ICF) or the extinction correction but only on the errors of measurement, which we have earlier estimated at a value of $20 \%$. Comparing the abundances of Chiappini et al. (2009) with our values we see the following. Both oxygen and nitrogen agree within the estimated errors. Our values of neon, sulfur and argon are higher in all three cases by a factor of about 2.5, clearly above the errors discussed above. In all three case the reason for this is the same: in many $\mathrm{PNe}$ the most important ions were not measured in the visual spectra $\left(\mathrm{Ne}^{+}, \mathrm{S}^{++}, \mathrm{S}^{+3}\right.$ and $\left.\mathrm{Ar}^{+}\right)$. All are measured in our infrared line spectra and therefore give more reliable results.

Consider now the chlorine abundance. In the visual spectra chlorine is represented by a single ion: $\mathrm{Cl}^{++}$. This ion has a weak doublet line at $\lambda 5517$ and $\lambda 5534$. These lines are difficult to measure accurately. Our infrared spectra sometimes also shows chlorine ions: $\mathrm{Cl}^{+}$is represented by a line at $14.37 \mu \mathrm{m}$ which has been seen in five of our nebulae. The $\mathrm{Cl}^{+3}$ line at $20.32 \mu \mathrm{m}$ has been seen twice. There is only enough information to determine the $\mathrm{Cl}$ abundance in about one half of the nebulae. The value is generally quite close to the solar chlorine abundance, but the chlorine abundance given by Chiappini et al. (2009) is a factor of 4 higher. The reason for this cannot be due to differences in measuring the chlorine line intensities because the same measurements were used by us as by Chiappini et al. (2009). 
A\&A 583, A71 (2015)
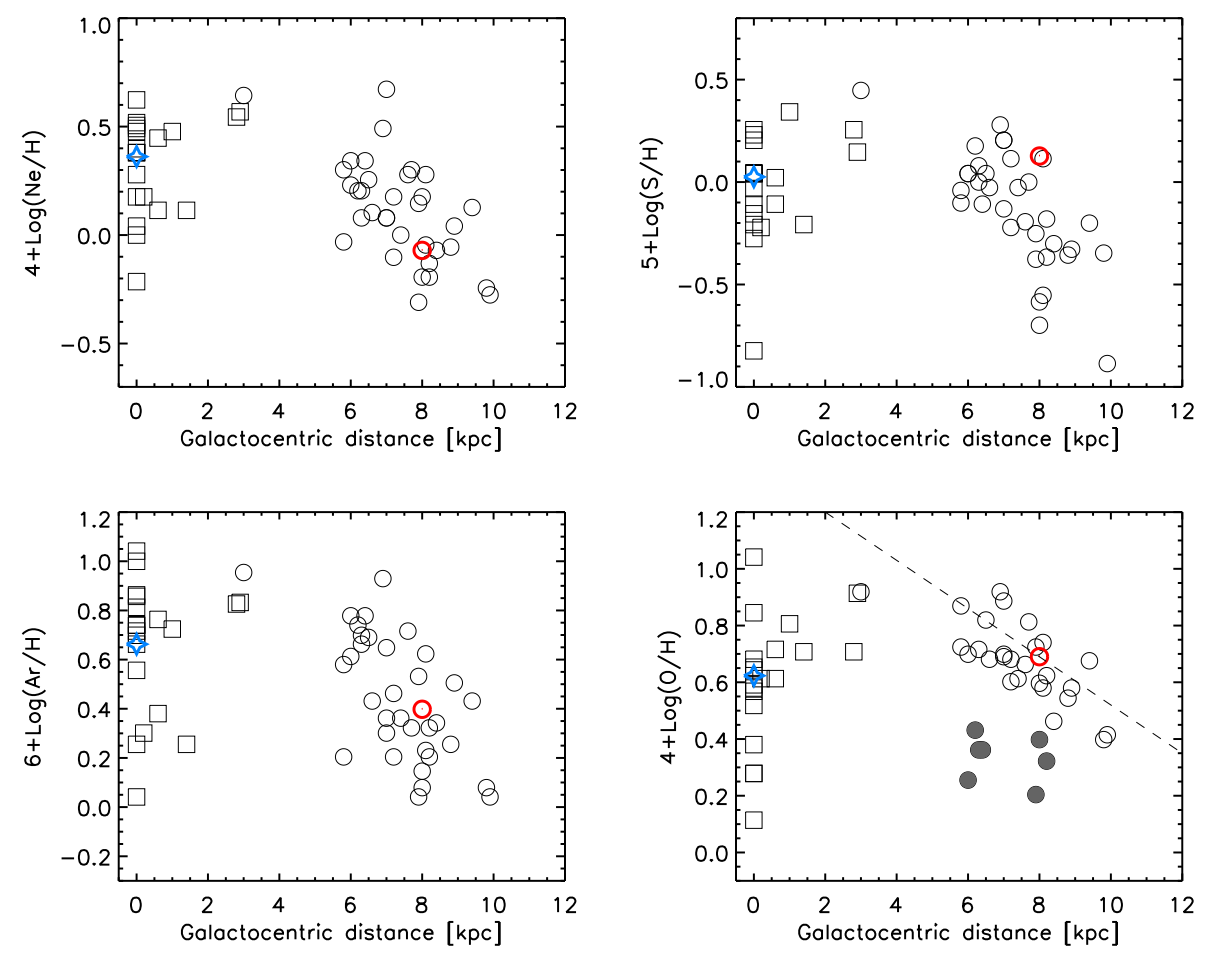

Fig. 2. Abundances of $\mathrm{Ne}, \mathrm{S}, \mathrm{Ar}$, and $\mathrm{O}$ as a function of distance from the Galactic centre. The bulge PNe (squares) do not have known distances but those with high velocity are all between 0 and $3 \mathrm{kpc}$ from the centre. For some of these PNe the distance listed by Gutenkunst et al. (2008) is used to avoid crowding of the points. The red and blue symbols indicate the solar abundances and the average bulge abundances respectively. The filled circles in the oxygen diagram indicate those $\mathrm{PNe}$ where oxygen burning is thought to have taken place.
The probable reason for the difference is the use of a different ICF although we cannot be certain of this because Chiappini et al. (2009) do not give the details of their determination. Our result is supported by other authors who have calculated the chlorine abundance: using essentially the same $\mathrm{Cl}$ III line intensity they obtain clearly lower $\mathrm{Cl}$ abundances. For example, in M3-21 Wang \& Liu (2007) found the chlorine abundance to be a factor of 14 lower than Chiappini et al. (2009), and in M3-17 Cuisinier et al. (2000) found the chlorine abundance to be a factor of 5.3 lower.

\section{Radial abundance gradient}

In this paper we confirm our earlier conclusion Gutenkunst et al. (2008) that the Bulge PNe abundances do not follow the Disc abundance gradient. The disc gradient was first seen in H II regions by Shaver et al. (1983). They noticed that the abundances of five elements showed a gradient between 4 and $14 \mathrm{kpc}$ from the Galactic centre. A gradient is also found for PNe in the same region of space, as has been discussed by Maciel et al. (2003) and Pottasch \& Bernard-Salas (2006). Maciel \& Costa (2009) have summarized the oxygen gradient separately. The regions closer to the Galactic centre are not discussed by these authors for two reasons. First the extinction in visible light in the bulge is large, difficult to measure and difficult to correct. Secondly the distance to a particular PN is often difficult to determine and hence its position in the Galactic plane is uncertain. Both papers show a straight line gradient of abundance of all elements considered as a function of distance from the Galactic centre. If one extends this line to the bulge the predicted abundances are (considerably) greater than those we have observed. This is illustrated in Fig. 2 where the individual abundances of four elements are plotted as functions of distance from the Galactic centre. The PNe abundances of the nebulae between 5 and $10 \mathrm{kpc}$ from the centre are taken from Pottasch \& Bernard-Salas (2010); the distance from the centre for these nebulae, taken from the same authors, is realistic. The abundances of the bulge PNe is taken from Table 7; the precise distance from the centre is difficult to determine but, as discussed above, their high radial velocity places them certainly within 2 or $3 \mathrm{kpc}$ from the Galactic centre. The diagrams for neon, argon and sulfur have several things in common. Firstly, the abundance gradient between 5 and $10 \mathrm{kpc}$ can be clearly seen. Secondly the bulge abundances for these elements are not an extension of this gradient although they do have a higher average value than the PNe between 5 and $10 \mathrm{kpc}$. Thus the abundance gradient appears to become much less steep closer to the centre, in agreement with the results of Gutenkunst et al. (2008). The diagram for oxygen is somewhat less similar to the other three elements. This has two origins. One is that hot-bottom burning has removed a substantial amount of oxygen in at least 6 of the PNe contributing to the gradient. That hot-bottom burning has occurred is seen by high values of N/O and $\mathrm{He} / \mathrm{H}$. The bulge $\mathrm{PNe}$ we are studying do not have values of $\mathrm{He} / \mathrm{H}$ so that we judge whether they have undergone hotbottom burning only on the basis of their N/O abundance ratio. Following the discussion of Pottasch \& Bernard-Salas (2010) and Karakas (2003) N/O should be greater than 0.8 if hot-bottom has converted a substantial amount of oxygen to nitrogen. Thus this process has occurred in PNG 002.6-3.4, PNG 004.9-49 and PNG 357.1-4.7. This seems to be confirmed by the fact that these three PNe have the lowest oxygen abundance of the bulge PNe (except for PNG 008.2+6.8 which as has been discussed has low abundances of all elements). Removing the oxygen abundance of these $\mathrm{PNe}$ from the average raises the average oxygen abundance in the bulge PNe from $4.2 \times 10^{-4}$ to $5.0 \times 10^{-4}$. The average oxygen abundance in the bulge is now slightly higher than is found further from the centre. We thus agree with Gutenkunst et al. (2008) that Galactic bulge abundances are substantially lower than predicted from the disc abundance gradient, although slightly higher than interstellar abundances at $8 \mathrm{kpc}$ from the centre.

The reason for the change in the bulge abundances is a matter of discussion (see Sellwood \& Binney 2002). It appears from the high velocities found in the bulge that these PNe are part of a bar structure. According to Sanchez-Blazquez (priv. comm.): "Numerical simulations have shown that non-axisymmetric 
S. R. Pottasch and J. Bernard-Salas: Abundances in the Galactic bulge

components, in particular bars, have strong gravitational torque which induce evolutionary processes". This has the effect of making the stars appear older with somewhat lower chemical abundances. This should be further studied.

\section{References}

Acker, A., Marcout, J., Ochsenbein, F., et al. 1992, Strasbourg-ESO catalogue (Garching: ESO)

Asplund, M., Grevesse, N., Sauval, A. J., et al. 2009, ARA\&A, 47, 481

Chiappini, C., Gorny, S. K., Stasinska, G., et al. 2009, A\&A, 494, 591

Cuisinier, F., Maciel, W. J., Koppen, J., et al. 2000, A\&A, 353, 543

Escudero, A. V., Costa, R. D. D., \& Maciel, W. J. 2004, A\&A, 414, 21

Exter, K. M., Barlow, M. J., \& Walton, N. A. 2004, MNRAS, 349, 1291

Gorny, S. K., Stasinska, G., Escudero, A. V., et al. 2004, A\&A, 427, 231

Gorny, S. K., Chiappini, C., Stasinska, G., et al. 2009, A\&A, 500, 1089

Gorny, S. K., Perea-Calderon, J. V., Garcia-Hernandez, D. A., et al. 2010, A\&A, 516, A39

Gutenkunst, S., Bernard-Salas, J., Pottasch, S. R., et al. 2008, ApJ, 660, 1206

Henry, R. B. C., Speck, A., Karakas, A., et al. 2012, ApJ, 749, 61

Houck, J. R., Appleton, P. N., Armus, L., et al. 2004, ApJS, 154, 18

Hummer, D. G., \& Storey, P. J. 1987, MNRAS, 224, 801

Karakas, A. I. 2003, Thesis, Monash Univ. Melbourne

Kingsburgh, R. L., \& Barlow, M. J. 1994, MNRAS, 271, 257
Lebouteiller, V., Bernard-Salas, J., Sloan, G. C., et al. 2010, PASP, 122, 231 Maciel, W. J., \& Costa, R. D. D. 2003, IAU Symp. 209 (Dopita \& Sutherland: Kwok), 351

Maciel, W. J., \& Costa, R. D. D. 2009, IAU Symp., 265, 317

Marigo, P., Bernard-Salas, J., Pottasch, S. R., et al. 2003, A\&A, 409, 619

Martín-Hernández, N. L., van der Hulst, \& Tielens, A. G. G. M. 2003, A\&A 407, 857

Mokiem, M. R., Martín-Hernández, N. L., Lenorzer, A., et al. 2004, A\&A, 419, 319

Perea-Calderon, J. V., Garcia-Hernandez, D. A., Garcia-Lario, P., et al. 2009, A\&A, 495, L5

Pottasch, S. R., \& Bernard-Salas, J. 2006, A\&A, 457, 189

Pottasch, S. R., \& Bernard-Salas, J. 2010, A\&A, 517, A95

Pottasch, S. R., \& Bernard-Salas, J. 2013, A\&A, 550, A35

Ratag, M. A. 1990, Thesis, Univ. Groningen

Rudolph, A. L., Fich, M., Bell, G. R., et al. 2006, ApJS, 162, 346

Sahai, R., Morris, M. R., \& Villar, G. G. 2011, AJ, 141, 134

Sellwood, J. A., \& Binney, J. J. 2002, MNRAS, 336, 785

Shaver, P. A., McGee, R.X, Newton, L. M., et al. 1983, MNRAS, 204, 53

Stanghellini, L., García-Hernández, D. A., García-Lario, P., et al. 2012, ApJ, 753,172

Surendiranath, R., \& Pottasch, S. R. 2008, A\&A, 483, 519

Surendiranath, R., Pottasch, S. R., \& García-Lario, P. 2004, A\&A, 421, 1051

Wang, W., \& Liu, X.-W. 2007, MNRAS, 381, 669

Webster, B. L. 1988, MNRAS, 230, 377 